Pathologe 2021 · 42 (Suppl 1):S69-S75 https://doi.org/10.1007/s00292-020-00897-3 Accepted: 16 December 2020

Published online: 15 March 2021

c) Springer Medizin Verlag GmbH, ein Teil von Springer Nature 2021

Guest editors

W. Roth, Mainz

P. Boor, Aachen

Saskia von Stillfried' $\cdot$ Till Acker $^{2,10} \cdot$ Martin Aepfelbacher $^{3} \cdot$ Gustavo Baretton $^{4,11}$. Roman David Bülow ${ }^{1} \cdot$ Karl-Friedrich Bürrig ${ }^{5,12}$. Hans-Ulrich Holtherm ${ }^{6}$. Danny Jonigk ${ }^{7} \cdot$ Ruth Knüchel $^{\prime} \cdot$ Raphael W. Majeed $^{8} \cdot$ Rainer Röhrig $^{8}$. Jan Wienströer ${ }^{8} \cdot$ Peter Boor ${ }^{1,9}$

${ }^{1}$ Institute of Pathology, University Hospital RWTH Aachen, Aachen, Germany

${ }^{2}$ Institute of Neuropathology, Justus-Liebig University Giessen, Giessen, Germany

${ }^{3}$ Institute for Medical Microbiology, Virology and Hygiene, University Medical Center HamburgEppendorf, Hamburg, Germany

${ }^{4}$ Institute of Pathology, University Hospital Carl Gustav Carus Dresden, Dresden, Germany

${ }^{5}$ Institute of Pathology Hildesheim, Hildesheim, Germany

${ }^{6}$ Federal Ministry of Health, Berlin, Germany

${ }^{7}$ Hannover Medical School, German Lung Research Center Biomedical Research in Endstage and Obstructive Lung Disease Hanover (BREATH), Hannover, Germany

${ }^{8}$ Institute for Medical Informatics, University Hospital RWTH Aachen University, Aachen, Germany

${ }^{9}$ Medical Clinic II (Nephrology and Immunology), University Hospital RWTH Aachen, Aachen, Germany

${ }^{10}$ German Society of Neuropathology and Neuroanatomy, Magdeburg, Germany

"German Society of Pathology, Berlin, Germany

${ }^{12}$ Federal Association of German Pathologists, Berlin, Germany

\title{
Cooperative approach of pathology and neuropathology in the COVID-19 pandemic
}

\section{German registry for COVID-19 autopsies (DeRegCOVID) and German network for autopsies in pandemics (DEFEAT PANDEMIcs)}

systematic and structured analysis of epidemiological data, biomaterial, and autopsy findings.

\section{Introduction}

The WHO declared the global outbreak of COVID-19 a pandemic on 11 March 2020. On September 7, the WHO Director-General stated, "This will not be the last pandemic. History teaches us that outbreaks and pandemics are a fact of life. But when the next pandemic comes, the world must be ready-more ready than it was this time" [8]. To cope with the consequences of the disease (coronavirus disease 2019, COVID-19) and pandemic caused by the severe acute respiratory syndrome coronavirus 2 (SARS-CoV-2) and to prepare for future pandemics, it is of utmost importance to mobilize and harmonize basic and applied medical research both nationally and globally [15], and to collect, analyze, and make the data available, if possible in a centralized manner. This is already happening in various registries, such as the LEOSS registry (Lean European Open Survey on SARS-CoV-2 infected patients) and the CAPACITYCOVID registry (registry of patients with COVID-19 including cardiovascular risks and complications).

) The autopsy is an essential tool for understanding disease pathogenesis of infectious diseases

An autopsy is an essential tool for understanding disease pathogenesis, espe- 


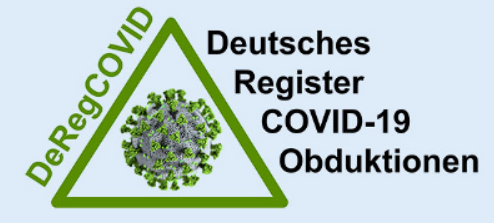

Fig. $1 \Delta$ Logo of DeRegCOVID (German Registry for COVID-19 Autopsies)

cially infectious diseases caused by new or so-called emerging or re-emerging viruses, e.g. influenza viruses, Ebola virus, hantaviruses, and coronaviruses (CoV), e.g., SARS-CoV [11], MERS-CoV or currently SARS-CoV-2 [19]. Already in the first published postmortem studies on COVID-19, the pathogenicity mechanisms underlying the particularly severe and fatal courses of the disease with potential therapeutic effects were identified. These organ-specific consequences of COVID-19 are discussed in detail in the corresponding articles in this special issue of the journal, especially for the lung and heart [2], kidney [3], or the central nervous system [14].

The recommendations for performing autopsies in Germany during the first wave of the SARS-CoV-2 pandemic ranged from the recommendation by the Robert Koch Institute to avoid autopsies due to occupational safety aspects, to a largely united position of German pathologists, forensic pathologists, and their professional societies for performing autopsies, for which there are many years of experience and guidelines based on other infectious diseases. The knowledge gained through autopsies made it clear to medical professionals, German health authorities, and the broader public that autopsies are an important and necessary tool for understanding the pathophysiology of COVID-19 and new or reemerging diseases in general. Autopsies can very quickly provide important information that can significantly improve the risk assessment, diagnosis, and treatment of patients. Various institutes of pathology have published recommendations or reports on the practical implementation of COVID-19 autopsies [4, 9, $10,13,16]$, which are also discussed in another article in this special issue [5].
To better manage the current pandemic, characterize the disease, gain new insights, and create preparedness structures for future epidemics and pandemics, it is necessary to establish central structures, such as a national autopsy registry and general procedural recommendations for conducting infectious autopsies, data collection, and biobanking in the event of a pandemic. We have established the first national registry for COVID-19 autopsies (DeRegCOVID). Extending this, we also launched the German Research Network for Pandemic Autopsies (DEFEAT PANDEMIcs) as part of the Network of University Medicine (NUM) to implement a harmonized data collection and systematic and standardized analysis of the suitability of the collected postmortem tissues and body fluids for virological, genomic, transcriptomic and imaging analyses on a nationwide level (https://www. netzwerk-universitaetsmedizin.de/).

\section{German registry for COVID-19 autopsies-DeRegCOVID}

Given the need for centralized and coordinated support, reporting, systematic biobanking, and structured data harmonization, we launched the first version of a Germany-wide registry of COVID-19 autopsies on April 15 (DeRegCOVID, - Fig. 1). Further information can be found on the website (www.DeRegCOVID.ukaachen.de) or inquired via e-mail (Covid.Pathologie@ ukaachen.de). The interdisciplinary team of DeRegCOVID currently consists of 13 employees from the Institute of Pathology (medical and scientific management), Institute of Medical Informatics (technical management), Clinical Trial Center Aachen (CTC-A, project management) as well as other employees from the Legal and Data Security Division of the University Hospital Aachen.

The main objective of this centralized national registry for COVID-19 autopsies is to collect, categorize, analyze and provide harmonized and factually anonymous data on all autopsies and related biomaterials of COVID-19 deceased persons in Germany to the medical and scientific community (• Fig. 2 , from [18]).
The DeRegCOVID is located at the University Hospital RWTH Aachen and was developed with the close support of the Federal Association of German Pathologists e. V. (BDP) and the German Society of Pathology (DGP). It is financed by the Federal Ministry of Health and has been positively reviewed by the Robert Koch Institute. Data collection is based on WHO recommendations and the German S1 guideline for performing autopsies. Many academic and nonacademic centers, including centers of the German Federal Armed Forces, are already involved in the registry, thus incorporating and implementing ethical and legal frameworks for all German autopsy centers.

Centers performing COVID-19 autopsies report epidemiological data (age, sex, time of death), data on preanalytical factors (time between death and autopsy), data on known clinical course, underlying diseases, findings and causes of death determined during the autopsy, and metadata on type and amount of locally archived tissue samples (e.g., organ/topography, FFPE, cryoasservation). Entry into the reporting system is done via the internet (https://covidpat. ukaachen.de/), using password-protected login data specifically set up for each center. The platform is constantly being optimized, especially following feedback from users, to further improve data entry. Experience has shown that, depending on the complexity of an autopsy case, it currently takes between 15 and $30 \mathrm{~min}$ to enter data. The staff of DeRegCOVID assists with all questions (Covid.Pathologie@ukaachen.de). The collected sample material remains with the respective centers (decentralized biobanking, - Fig. 2a). The DeRegCOVID follows the principle that all centers retain the rights to their reported data and materials.

\section{) All centers retain the rights to their reported data and materials}

For data reporting, each center must comply with local regulatory requirements, especially regarding ethical and data protection issues. DeRegCOVID 
Pathologe 2021 - 42 (Suppl 1):S69-S75 https://doi.org/10.1007/s00292-020-00897-3

(c) Springer Medizin Verlag GmbH, ein Teil von Springer Nature 2021

S. von Stillfried · T. Acker · M. Aepfelbacher · G. Baretton · R. D. Bülow · K.-F. Bürrig · H.-U. Holtherm · D. Jonigk · R. Knüchel · R. W. Majeed • R. Röhrig ·. Wienströer $\cdot$ P. Boor

\section{Cooperative approach of pathology and neuropathology in the COVID-19 pandemic. German registry for COVID-19 autopsies (DeRegCOVID) and German network for autopsies in pandemics (DEFEAT PANDEMICS)}

\section{Abstract}

Background. Autopsy is an important tool for understanding the pathogenesis of diseases, including COVID-19.

Material and methods. On 15 April 2020, together with the German Society of Pathology and the Federal Association of German Pathologists, the German Registry of COVID-19 Autopsies (DeRegCOVID) was launched (www.DeRegCOVID.ukaachen.de). Building on this, the German Network for Autopsies in Pandemics (DEFEAT PANDEMIcs) was established on 1 September 2020.

Results. The main goal of DeRegCOVID is to collect and distribute de facto anonymized data on potentially all autopsies of people who have died from COVID-19 in Germany in order to meet the need for centralized, coordinated, and structured data collection and reporting during the pandemic. The success of the registry strongly depends on the willingness of the respective centers to report the data, which has developed very positively so far and requires special thanks to all participating centers. The rights to own data and biomaterials (stored decentrally) remain with each respective center. The DEFEAT PANDEMIcs network expands on this and aims to strengthen harmonization and standardization as well as nationwide implementation and cooperation in the field of pandemic autopsies.
Conclusions. The extraordinary cooperation in the field of autopsies in Germany during the COVID-19 pandemic is impressively demonstrated by the establishment of DeRegCOVID, the merger of the registry of neuropathology (CNS-COVID19) with DeRegCOVID and the establishment of the autopsy network DEFEAT PANDEMlcs. It gives a strong signal for the necessity, readiness, and expertise to jointly help manage current and future pandemics by autopsy-derived knowledge.

Keywords Autopsy · Interoperability · Registries · SARSCoV-2 Tissue banks

\section{Kooperatives Vorgehen der Pathologie und Neuropathologie in der COVID-19-Pandemie. Deutsches Register für COVID-19-Obduktionen (DeRegCOVID) und DeutschesNetzwerk für Autopsien in Pandemien (DEFEAT PANDEMIcs)}

\section{Zusammenfassung}

Hintergrund. Die Obduktion ist ein wichtiges Instrument zum Verständnis der Pathogenese von Krankheiten, inklusive COVID-19.

Material und Methoden. Am 15.04.2020 wurde zusammen mit der Deutschen Gesellschaft für Pathologie und dem Bundesverband der deutschen Pathologen das Deutsche Register von COVID-19-Obduktionen (DeRegCOVID) gestartet (www.DeRegCOVID.ukaachen.de) und darauf aufbauend seit 01.09.2020 das Deutsche Netzwerk für Autopsien in Pandemien (DEFEAT PANDEMIcs) etabliert. Ergebnisse. Hauptziel des DeRegCOVID ist es, faktisch anonymisierte Daten über idealerweise alle Obduktionen von COVID-19Verstorbenen in Deutschland zu sammeln und zur Verfügung zu stellen, um dem Bedarf an zentralisierter, koordinierter und strukturierter Datenerhebung und Berichterstattung in der Pandemie gerecht zu werden. Der Erfolg des Registers hängt von der Bereitschaft der jeweiligen Zentren zur Meldung der Daten ab. Diese hat sich bislang sehr positiv entwickelt und wir danken allen beteiligten Zentren. Dabei verbleiben die Rechte an eigenen Daten (und dezentral verbleibenden Biomaterialen) bei den jeweiligen Institutionen. Das Netzwerk DEFEAT PANDEMIcs zielt auf eine Verstärkung der Harmonisierung und Standardisierung sowie die bundesweite Implementation und Kooperation im Bereich von Pandemieobduktionen.

Schlussfolgerungen. Die außerordentliche Kooperation in Deutschland im Bereich der Obduktionen während der COVID19-Pandemie ist durch den Aufbau des DeRegCOVID, dessen Fusionierung mit dem Register der Neuropathologie (CNSCOVID19) und dem Aufbau des Netzwerks DEFEAT PANDEMIcs eindrücklich belegt. Es ist ein starkes Signal für die Notwendigkeit, Bereitschaft und Expertise, mit durch Autopsie gewonnenen Erkenntnissen zur gemeinsamen Bewältigung gegenwärtiger und künftiger Pandemien beizutragen.

\section{Schlüsselwörter}

Autopsie · Interoperabilität · Register · SARSCoV-2.Gewebebanken actively supports the participating centers in meeting the local regulatory requirements.

After curation and harmonization, the available data are centrally evaluated and reported to the medical and scientific community: to the Professional Association of Pathologists and Neuropathologists (Bundesverband
Deutscher Pathologen e.V. [BDP], the German Society of Pathology [DGP], the German Society of Neuropathology and Neuroanatomy [DGNN]), the Federal Ministry of Health and the Robert Koch Institute, and in coordination with these, to the public.

An important aspect and a central task of DeRegCOVID is the implementation of interfaces to national and international data platforms. The reporting centers will provide their centrally curated data in a syntactically and semantically standardized and annotated form so that they can then be uploaded into national and international research projects. One such example is the research data platform of the National Pandemic Cohort 


\section{Schwerpunkt: COVID-19}

German registry for COVID-19 autopsies: DeRegCOVID

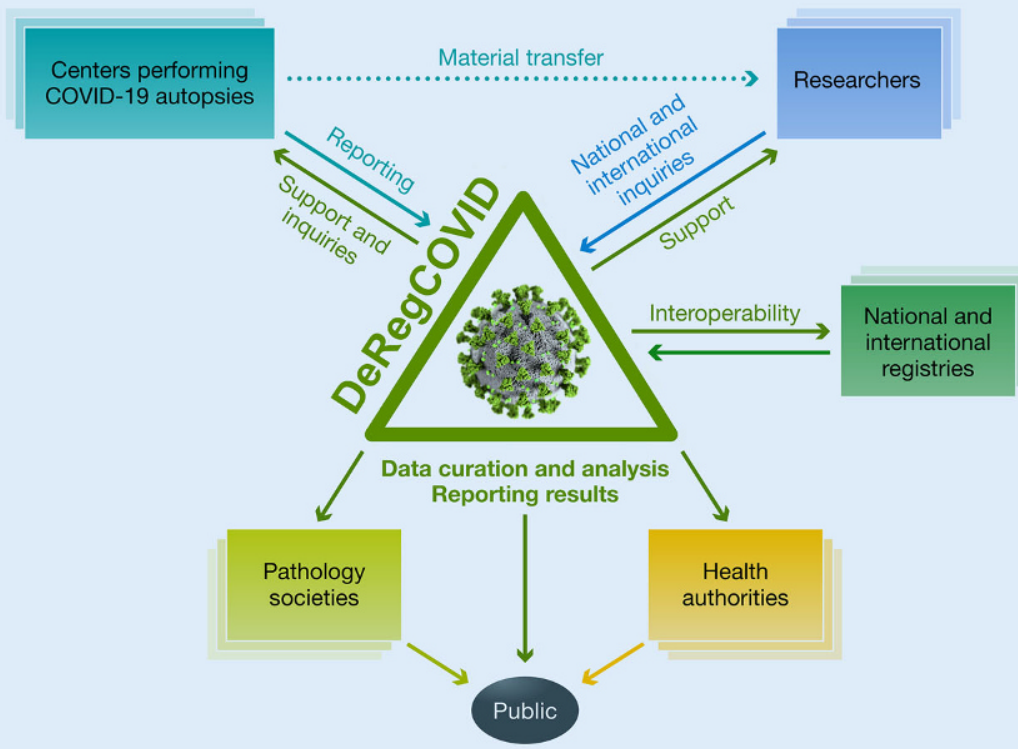

\begin{tabular}{l|l} 
Launch v.1 15 April $2020 \quad$ Approval by ethics committee of university hospital (RWTH Aachen)
\end{tabular} Launch v.2 29 April 2020 No. EK 092/20
Data on available postmortem biosamples

from more than 25 tissues and organs



Data collected from deceased who had been diagnosed with COVID-19 include

- demographic data,

- known preexisting conditions,

- course of disease,

b pathological diagnosis and cause of death.

Registered at Clinical Trial Center Aachen (CTCA) No. EK 20-096

Fig. 2 A Tasks and objectives of DeRegCOVID (German Registry for COVID-19 Autopsies), with kind permission from von Stillfried et al. [18]

Network(NAPKON) within the Network of University Medicine (NUM; https:// www.netzwerk-universitaetsmedizin. $\mathrm{de} /$ ). Furthermore, interfaces to the structures of the registries/biobanks within the German Health Centers, e.g., German Center for Lung Research (Deutsches Zentrum für Lungenforschung, DZL), are planned. For this purpose, data set definition and export interfaces will be continuously adapted to the evolving definition of the GECCO data set (German Corona Consensus data set). Cooperation with international initiatives is also planned.

\section{》) Implementation of interfaces to national and international data platforms}

In addition to the core tasks of central data acquisition and reporting, DeRegCOVID fulfills several other tasks. These include, for example, support in questions concerning practical aspects of COVID-19 autopsies, as a central source of information for procedural instructions and recommendations, e.g., safety measures and occupational health and safety in the context of autopsies or biobanking of biomaterial of COVID19 deceased persons.

Another important task is to serve as a mediator between participating centers and national or international scientists. If scientists require an autopsy biomaterial or data for a study, they can send a request to DeRegCOVID (an application form is available at www. DeRegCOVID.ukaachen.de). The team of DeRegCOVID first checks the plausibility of the request, i.e., whether the project can be answered by the available biomaterial or data from the registry. Our initial experience shows that some requests cannot be served, given that the questions cannot be answered with the proposed methods on postmortem biomaterial and available data. In case of suitable inquiries, the centers with available biomaterials and data are identified and contract between the requesting researchers and the reporting centers and biobanks with appropriate material is established (a function of "scientific Tinder"). Thus, it is possible to identify cases in a specific disease stage (e.g., particularly early or late stage), with a specific pathological diseases pattern (e.g., hemophagocytic lymphohistiocytosis), with specific comorbidities (e.g., diabetes, kidney disease) or therapies (e.g., no therapy, long-term or intensive therapy, ECMO). This also facilitates multicenter studies that include a sufficiently large number of cases and to produce robust results. The first example, which was supported by DeRegCOVID, is the work describing the pathological and molecular characteristics of pulmonary involvement in COVID-19 based on autopsies, published in the New England Journal of Medicine [1]. Further studies have been published in the meantime $[6,7,12]$, and some are under review or in preparation. 


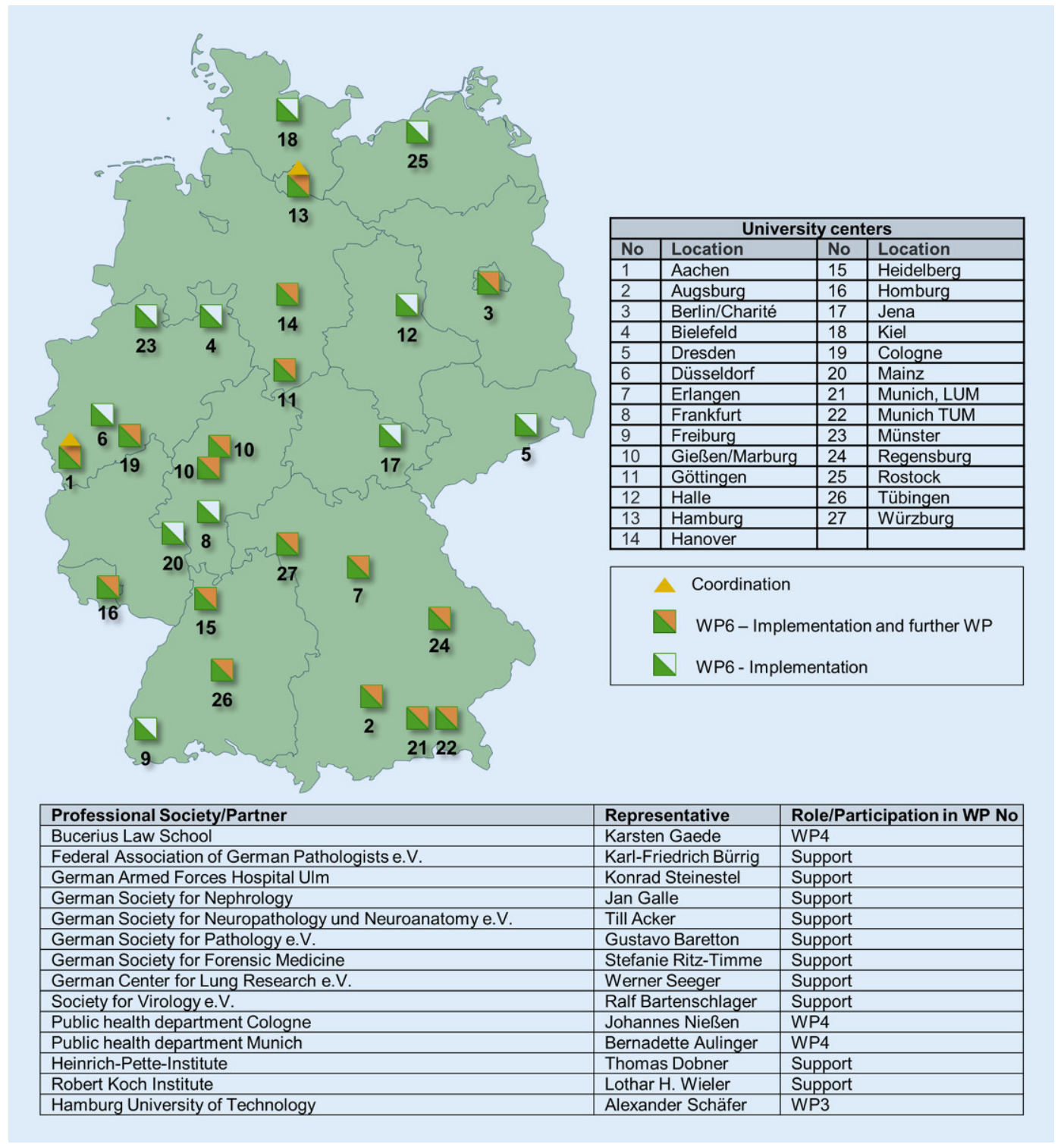

Fig. $3 \triangleleft$ Participating university centers, profes sional societies, and partners of DEFEAT PANDEMIcs. WP work packages (Map source: Portal of the statistical offices of the Federal and State Governments (DeStatis); David Liuzzo. This file is licensed under the Creative Commons Attribution-Share Alike 2.0 license Germany.)
Compartment-specific detection of the virus in the tissues of COVID-19, but often also the basic confirmation of SARS-CoV-2 infection in deceased persons, are important aspects in the evaluation of autopsy results. Therefore another task of DeRegCOVID is to support the detection of SARS-CoV-2 in tissues for which different methods are available. Method-specific advantages and disadvantages and indications of the different methods are discussed in another article in this issue [17].

\section{German network for pandemic autopsies-DEFEAT PANDEMIcs}

As a consequence of the work described above, which has shown the importance of autopsies for understanding COVID19, the German Research Network for Autopsies in Pandemics was founded (DEFEAT PANDEMIcs).

The DEFEAT PANDEMIcs network currently consists of 27 university centers (with more than 50 pathological, neuropathological, and forensic medicine institutions) and 14 non-university research institutions, including the Robert Koch Institute (• Fig. 3). It is funded by the German Federal Ministry of Education and Research within the framework of the Network of University Medicine (NUM; https://www.networkuniversitaetsmedizin.de/). The goal of the DEFEAT PANDEMIcs network is to implement and further improve a systematic, structured, harmonized, comprehensive, and rapid analysis of epidemiological data, findings, and tissue samples from autopsies during the COVID-19 pandemic and future pandemics or epidemics on a nationwide level.

DeRegCOVID is the central and sustainable data platform, the information broker, and thus the electronic backbone of the DEFEAT PANDEMIcs network. Due to the modular and thus scalable architecture of the registry, 


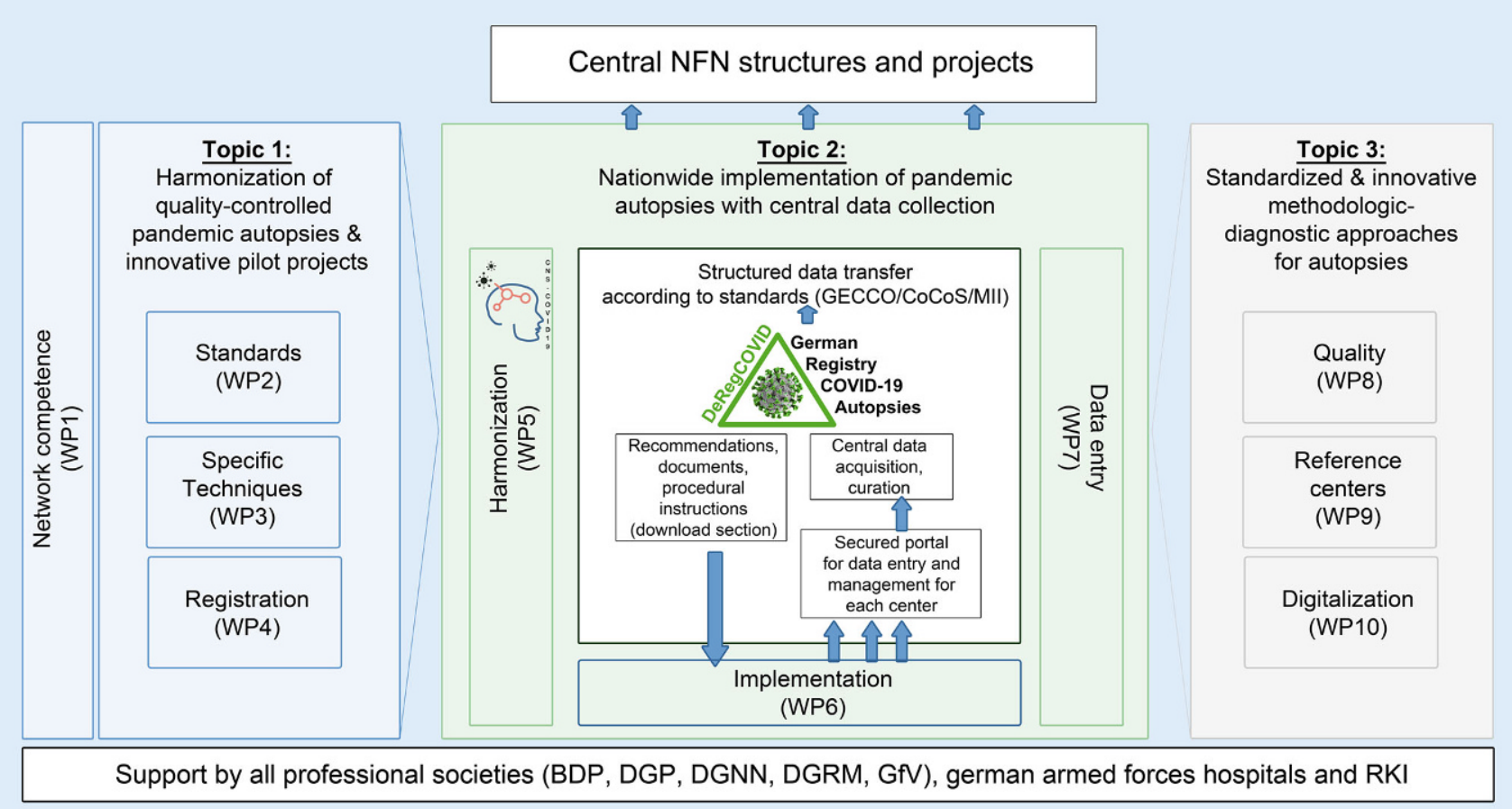

Fig. 4 ム Topics and tasks of DEFEAT PANDEMICs. BDP Federal Association of German Pathologists, DGNN German Society for Neuropathology and Neuroanatomy, DGP German Society for Pathology, DGRM German Society for Forensic Medicine, GfVSociety for Virology, NFN German Research Network, RKI Robert Koch Institute

DeRegCOVID can be supplemented by any number of centers. All functions of DeRegCOVID are also provided for the entire network. The Germanywide registry CNS-COVID19 (www. cns-covid19.de), founded by the German Society for Neuropathology and Neuroanatomy (DGNN), was merged with DeRegCOVID within the framework of DEFEAT PANDEMIcs. The registry CNS-COVID19, located at the JustusLiebig University of Giessen, supported the systematic research of central and peripheral nervous system involvement in COVID-19 via standardized sampling and decentralized biobanking of human tissue samples from defined CNS/PNS/ muscle areas in COVID-19 autopsies and is supported by more than 35 university and non-university neuropathology and pathology institutions.

The tasks of DEFEAT PANDEMIcs cover three subject areas (see also - Fig. 4):

1. Harmonization of quality-assured autopsies with decentralized sample storage and innovative pilot projects for pandemic autopsies, with the work packages (WP):
- WP1: Network competence and coordination,

- WP2: Preparation of recommendations, process standards, quality management, and emergency plans for postmortem examinations in case of a pandemic,

- WP3: Study on the implementation and validation of specific autopsy techniques (e.g., minimally invasive autopsy, postmortem imaging),

- WP4: Pilot study on national mortality registration through autopsies in case of a pandemic following the Infection Protection Act.

2. Nationwide implementation of pandemic autopsies with centralized dissemination and data collection:

- WP5: Harmonization of existing structures into the central data registry for COVID-19 autopsies (DeRegCOVID),

- WP6: Germany-wide implementation of autopsies and biobanking,

- WP7: Measures to increase central data reporting of autopsy results.
3. Standardized and innovative methodological-diagnostic approaches to autopsies:

- WP8: Sample quality requirements for analytical use of autopsy samples,

- WP9: Organ-specific COVID-19 pathology reference centers.

\section{Conclusions}

An early, unified public relations effort by German pathologists and pathological societies made it clear to medical professionals, German health authorities, and the public that autopsies remain an essential tool for understanding the pathophysiology of emerging diseases in general and of COVID-19 in particular. This was also demonstrated by the important and already early autopsy-based medical-scientific work, many of which originated from the German-speaking countries and which in part led to changes in therapeutic strategies for severe COVID19 courses. The collaborative approach was further strengthened at a very early stage by the establishment of the centralized national registry for COVID-19 
autopsies (DeRegCOVID) and is being further developed with the German Research Network for Pandemic Autopsies (DEFEAT PANDEMIcs). These sustainable structures will help us to jointly manage the current pandemic as well as future epi- and pandemics.

\section{Conclusion for practice}

- The German Registry for COVID-

19 Autopsies (DeRegCOVID; www.

DeRegCOVID.ukaachen.de):

- Collects data centrally and electronically of ideally all COVID-19 autopsies in Germany.

- Any institute or center can participate.

- Supports all centers and researchers.

- The data sovereignty and all biomaterials remain with the respective institutes or centers.

- Analyzes and reports on collected data in cooperation with the participating centers.

- Acts as the electronic backbone and sustainable structure for the DEFEAT PANDEMIcs network.

- The German Network for Autopsies in Pandemics (DEFEAT PANDEMIcs):

- Aims at a nationwide implementation of high-quality, standardized, and harmonized data collection and biobanking of autopsies in pandemics.

- Is a network of 27 university hospitals with more than 60 participating institutes (pathology, neuropathology, and forensic medicine) and 14 associated societies and partners.

- Serves as a preparedness structure for future pandemics.

\section{Corresponding address}

Saskia von Stillfried

Institute of Pathology, University Hospital RWTH Aachen

Pauwelsstr. 30, 52074 Aachen, Germany

svonstillfri@ukaachen.de

Peter Boor
Institute of Pathology,
University Hospital RWTH
Aachen
Pauwelsstr. 30, 52074 Aachen,
Germany
pboor@ukaachen.de

Acknowledgements. The authors would like to thank all centers and institutes that perform COVID19 autopsies and participate in DeRegCOVID and DEFEAT PANDEMIcs. Our thanks also apply to the many involved coworkers of the University Clinic of the RWTH Aachen, who helped to develop, further optimize, and maintain DeRegCOVID. The authors would especially like to thank the Dean of the Medical Faculty of the RWTH Aachen University, Univ.Prof. Dr. Stephan Uhlig, as well as the staff of the CTC-A (Clinical Trial Center Aachen) Jana Böcker, Jens Schmidt and Pauline Tholen, and the Legal Department, Sarah Allen, and Cora Savelsberg. This work was supported by the Federal Ministry of Health (ZMVI1-2520COR201) and the Federal Ministry of Education and Research within the framework of the Network of University Medicine (DEFEAT PANDEMIcs, 01KX2021) and by START 125/17.

\section{Declarations}

Conflict of interest. S. v. Stillfried, T. Acker, M. Aepfelbacher, G. Baretton, R.D. Bülow, K.-F. Bürrig, H.-U. Holtherm, D. Jonigk, R. Knüchel, R.W. Majeed, R. Röhrig, J. Wienströer and P. Boor declare that they have no competing interests.

For this article no studies with human participants or animals were performed by any of the authors. All studies mentioned were in accordance with the ethical standards indicated in each case.

\section{References}

1. Ackermann M, Verleden SE, Kuehnel M et al (2020) Pulmonary Vascular Endothelialitis, Thrombosis, and Angiogenesis in Covid-19. N Engl J Med 383(2):120-128

2. Ackermann $M$, Werlein $C$, Länger $F$ et al (2021) COVID-19: effects on the lungs and heart. Pathologe. https://doi.org/10.1007/s00292-02100918-9

3. Amann K, Boor P, Wiech T et al (2021) COVID-19 effects on the kidney. Pathologe. https://doi.org/ 10.1007/s00292-020-00900-x

4. Barton LM, Duval EJ, Stroberg E et al (2020) COVID19 autopsies, Oklahoma, USA. Am J Clin Pathol 153:725-733

5. Boor P, Eichhorn P, Hartmann A et al (2021) Practical aspects of COVID-19 autopsies. Pathologe. https:// doi.org/10.1007/s00292-021-00925-w
6. Fuest M, Boor P, Knuechel Retal (2020) Postmortem conjunctival and nasopharyngeal swabs in SARSCoV-2 infected and uninfected patients. Acta Ophthalmol. https://doi.org/10.1111/aos.14559

7. Gagiannis D, Steinestel J, Hackenbroch C et al (2020) Clinical, serological, and histopathological similarities between severe COVID-19 and acute exacerbation of connective tissue diseaseassociated interstitial lung disease (CTD-ILD). Front Immunol 11:587517

8. Ghebreyesus TA (2020) WHO Director-General's opening remarks at the media briefing on COVID-19-7 September 2020. https://www. who.int/director-general/speeches/detail/whodirector-general-s-opening-remarks-at-themedia-briefing-on-covid-19---7-september2020. Lastaccess: 11.11 .2020

9. Hanley B, Lucas SB, Youd E et al (2020) Autopsy in suspected COVID-19 cases. J Clin Pathol 73:239-242

10. Lacy JM, Brooks EG, Akers J et al (2020) Covid19: postmortem diagnostic and biosafety considerations. Am J Forensic Med Pathol 41(3):143-151

11. Nicholls JM, Poon LLM, Lee KC et al (2003) Lung pathology of fatal severe acute respiratory syndrome. Lancet 361:1773-1778

12. Nicolai L, Leunig A, Sophia B et al (2020) Vascular neutrophilic inflammation and immunothrombosis distinguish severe COVID-19 from influenza pneumonia. J Thromb Haemost. https://doi.org/ 10.1111/jth.15179

13. Parkash V, Smith SM (2020) Risk assessment of autopsy-acquired SARS-coV-2 Coronavirus (COVID-19). Arch Pathol Lab Med. https://doi.org/ 10.5858/arpa.2020-0500-LE

14. Ritschel N, Radbruch H, Herden C et al (2021) COVID-19 and the central and peripheral nervous system. Pathologe. https://doi.org/10.1007/ s00292-021-00924- $x$

15. Sansonetti PJ (2020) COVID-19, chronicle of an expected pandemic. EMBO Mol Med 12:e12463

16. Sperhake JP (2020) Autopsies of COVID-19 deceased? Absolutely! Leg Med 47:101769

17. Von Stillfried S, Boor P (2021) Methods of SARSCoV-2 detection in tissue. Pathologe. https://doi. org/10.1007/s00292-021-00919-8

18. Von Stillfried S, Bulow RD, Rohrig R et al (2020) Autopsy registry can facilitate COVID-19 research. EMBO Mol Med 12:e12885

19. Wang D, Hu B, Hu C et al (2020) Clinical characteristics of 138 hospitalized patients with 2019 novel Coronavirus-infected pneumonia in Wuhan, China. JAMA 323(11):1061-1069 